\title{
Análisis de Necropsias de la Sección de Infecciosos de un Servicio de Pediatría. Hospital "Guillermo Grant Benavente", Concepción
}

Dres. S. Torche*, E. Toro*, H. Suárez**, L. Román**

La necropsia es un procedimiento de estudio de gran utilidad para el médico, pues permite relacionar los hechos clínicos con los hallazgos anatomopatológicos, desempeña un papel impor. tante en la corrección y control de las causas de muerte, ayuda a discernir los casos en que se llega a la mesa de autopsia sin un diagnóstico preciso y complementa la experiencia del médico, que la puede aplicar en beneficio de otros pacientes.

Una correlación entre patología infecciosa y hallazgos anatomopatológicos es escasa en la literatura, lo cual nos motivó a realizar este estudio.

\section{MATERIAL Y METODO}

En la revisión del material llama la atención el bajo porcentaje de pacientes que tuvo estudio anatomopatológico, que corresponde al $29,1 \%$ del total de fallecidos en el período estudiado. Esto se explica porque a pesar de ser el Hospital Guillermo Grant Benavente un hospital clinico regional de importancia, no cuenta con un servicio propio de Anatomía Patológica, y debe solicitar prestación de servicio a la Universidad, con las consiguientes limitaciones que esto implica.

Se revisaron las historias clínicas e informes anatomopatológicos de 63 niños fallecidos entre enero de 1972 y diciembre de 1974, provenientes de la Sección Infecciosos del Servicio de Pediatría del Hospital Gmo. Grant Benavente, de Concepción.

\footnotetext{
* Médicos Residentes Becarios, Iepartamento de Pediatria, Universidad de Concepción.

* Sección Infecciosos, Servicio de Pediatría, Hospital Guillermo Grant Benavente, Concepción.
}

Se analizaron en las historias clínicas: edad, sexo, estado de nutrición, procedencia, diagnóstico de ingreso, estudio clínico y de laboratorio, diagnóstico con que se envía a autopsia, principal diagnóstico de necropsia y otros hechos que se consideraron de interés.

Se eligió la patología infecciosa debido a la agudeza y gravedad de los cuadros clínicos, ya que en muchas oportunidades el fallecimiento de estos pacientes puede ser evitado mediante un manejo adecuado.

\section{RESULTAIOOS}

En el período estudiado ingresaron a la Sección Infecciosos 2.916 pacientes. De éstos fallecieron 216, lo que representa una mortalidad global del $7,4 \%$. Por la limitación señalada anteriormente se efectuaron sólo 63 necropsias, que constituyen el material analizado (tabla 1).

Tabla 1

DISTRIBUCION DE INGRESOS Y FALLECIDOS DE 2.916 NIÑOS ENTRE LOS AÑOS 1972-1974 UNIDAD INFECCIOSOS. H.C.R.C.

\begin{tabular}{lrrrrr}
\multirow{2}{*}{ Años } & Ingreso & \multicolumn{2}{c}{ Fallecidos } & \multicolumn{2}{c}{ Autopsiados } \\
& & Casos & $\%$ & Casos & $\%$ \\
\cline { 3 - 6 } 1972 & 754 & $\mathbf{8 3}$ & 11,0 & 14 & 16,8 \\
1973 & 763 & 49 & 6,4 & 13 & 26,5 \\
1974 & 1.399 & $\mathbf{8 4}$ & $\mathbf{6 , 0}$ & 36 & $\mathbf{4 2 , 8}$ \\
\hdashline Total & 2.916 & 216 & 7,4 & 63 & 29,1 \\
\hline
\end{tabular}

La distribución por sexo fue la siguiente: 44 hombres $(73 \%)$ y 19 mujeres $(27 \%)$.

Distribución por edad: el $54 \%$ de los fallecidos está constituido por lactantes (34 casos) 
dentro de los cuales 20 eran niños menores de 6 meses. Cabe destacar que en el 73\% (46 casos) las edades fluctuaban entre 0 y 4 años (figura 1).

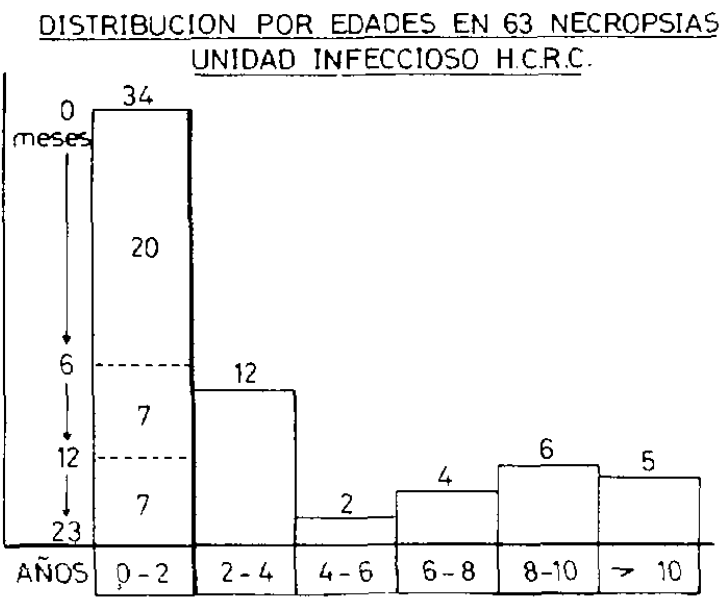

Fig. 1

Estado nutritivo: al analizar el estado de nutrición en el grupo de lactantes (34 casos) se encontró un $67 \%$ con déficit ponderal (figura 2).

\section{ESTADO NUTRICIONAL DE 34 LACTANTES}

AUTOPSIADOS. UNIDAD INFECCIOSO

\section{DESNUTRIDOS}

\section{H.C.R.C.}

$67 \%$

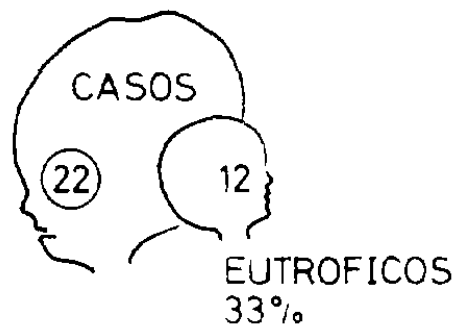

Fig. 2

Promedio de días de hospitalización: la mitad de los niños (32 casos) falleció dentro de las primeras 48 horas del ingreso y el $70 \%$ dentro de los primeros 4 dias de hospitalización.

Se estudió la procedencia de los niños, ya que el hospital, dada su calidad de Base de Area. recibe gran parte de sus pacientes de hospitales periféricos. El $50 \%$ provenía de otras localidades; de éstos, la gran mayoría permaneció hospitalizada uno o más días en esos centros previo a su traslado.

Se destaca que la mitad de los fallecidos dentro de las primeras 48 horas procedía de hospitales periféricos, ingresando en grave estado (Fig. 3).
PROCEDENCIA DE 63 PACIENTES AUTOPSIADOS ENTRE 1972-1974. UNIDAD INFECCIOSO H.C.R.C.

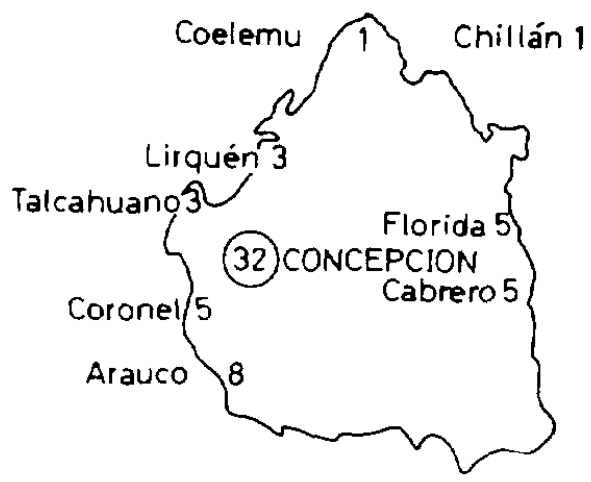

Fig. 3

Localización de la patología infecciosa que determinó la muerte: para una mayor comprensión, se agrupó la patología infecciosa por sistemas. La patología del Sistema Nervioso ocupó la primera causa de muerte con un $40 \%$ (25 casos), seguido del Aparato Respiratorio con un 38\% (24 casos) (Fig. 4).

\section{DISTRIBUCION POR SISTEMAS}

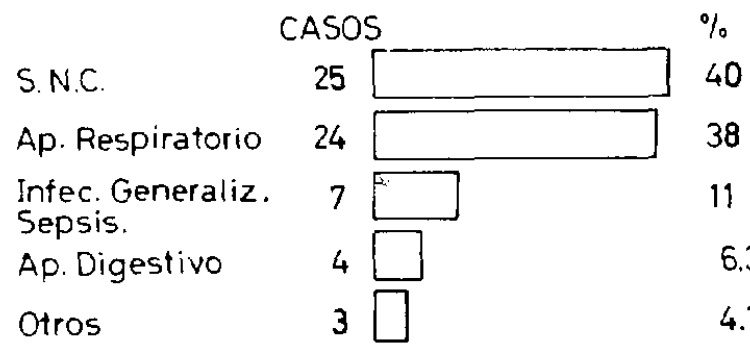

Fig. 4

Patolugia infecciosa del Sistema Nernoso

Este grupo lo conforman 25 pacientes, cuya patología se apreciá en la figura 5 .

OISTRIBUCION 24 CASOS CON PATOLOGIA APARATO RESPIRATORIO FALLECIDOS EN LA UNIDAD INFECCIOSO HCRC. ENTRE 1972-1974

CASOS $\%$

Complicaciones sarampión $12 \square 50$

Difteria

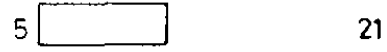

L.T.B.A

3

13

Tétano

2

Bronquiolitis

$1 \square$

8

Cuerpo extraño en tráquea $\%$ 8

. 
La patología más frecuente es la meningitis supurada. El $90 \%$ de los fallecidos por esta causa eran lactantes. Lo más relevante de los hallazgos anatomopatológicos lo constituye la asociación con otitis media supurada uni o bilateral en 7 casos, en los cuales el examen otorrinológico no había revelado alteraciones en vida. En 8 casos el diagnóstico de ingreso fue concordante con el diagnóstico anatomopatológico. En los 4 casos restantes no se hizo el diagnóstico al ingreso según se observa en la figura 8 . En los 2 niños cuyo diagnóstico de ingreso fue bronconeumonía se corrigió el diagnóstico antes de 48 horas. En cambio, los 2 pacientes que ingresaron como diarrea aguda, permanecieron hospitalizados por más de una semana en la Sección Lactantes, antes de efectuar el diagnóstico de meningitis (Fig. 6).

RELACION ENTRE DIAGNOSTICO DE INGRESO Y ANATOMOPATOLOGICO EN 12 CASOS DE MENINGITIS

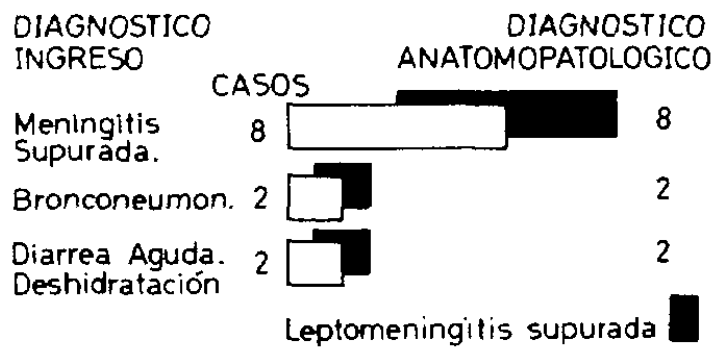

Fig. 6

El segundo grupo lo constituye la meningoencefalitis vírica; en ellas, el hecho antomopatológico más importante se refiere a la asociación con bronconeumonía paravertebral o por aspiración en todos los informes de necropsia.

El tercer grupo lo constituye la meningitis tuberculosa. En todos el diagnóstico fue hecho en forma tardía, incluso en uno el diagnóstico se hizo sólo por la necropsia y corresponde al único lactante del grupo. El informe anatomopatológico reveló concomitancia de tuberculosis pulmonar en todos ellos.

El cuarto grupo está representado por tres casos de hemorragia cerebral. Todos fueron considerados como meningitis supurada en algún momento de su evolución, interpretándose como traumáticas las punciones lumbares con LCR hemorrágico.

En un paciente portador de méduloblastoma, el estudio clínico y de laboratorio orientaban hacia una meningitis tuberculosa y sólo la necropsia reveló la verdadera naturaleza del padecimiento.

\section{Patologia infecciosa del Aparato Respiratono (Fig. 7).}

DISTRIBUCION 25 CASOS CON PATOLOGIA DEL SISTEMA NERVIOSO FALLECIDOS EN LA UNIDAD INFECCIOSO HCRC ENTRE 1972-1974

\begin{tabular}{|c|c|c|}
\hline \multicolumn{2}{|c|}{ CASOS } & $\%$ \\
\hline Meningitis supurada & 12 & 48 \\
\hline Meningoencefalitis vir & 4 & 16 \\
\hline Meningitis T.BC. & 4 & 16 \\
\hline Hernorragia cerebral & 3 & 12 \\
\hline Meduloblastoma & 1 & 4 \\
\hline Hidrocefalia & 1[ & 4 \\
\hline
\end{tabular}

Fig. 7

La mitad de la patología del aparato respiratorio corresponde a complicaciones del sarampión. Las tres cuartas partes de los casos presentaron laringotraqueobronquitis aguda (LTBA) sarampionosa grave, siendo necesaria traqueostomía de urgencia. Todos presentaron bronconeumonia de sobreinfección (Fig. 8).

\section{COMPLICACIONES 12 CASOS SARAMPION}

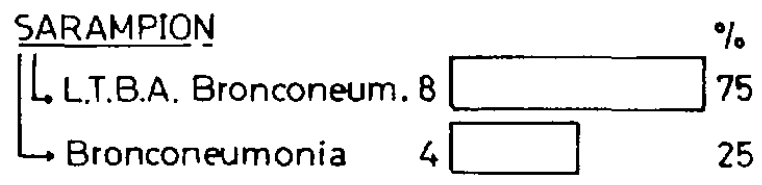

Fig. 8

Se encontraron 5 difterias, las que tenían en común los siguientes hechos: el tipo maligno, la procedencia rural, la falta de vacunación y el ingreso tardío. En cuatro coexistían miocarditis clínica y anatomopatológica.

En el rubro de las LTBA, se destaca que la traqueostomía se indicó en forma oportuna y los pacientes fallecieron por complicación infecciosa respiratoria aguda.

El cuarto rubro está representado por dos tétanos en recién nacidos que tienen en común: la procedencia rural, el ingreso tardío y la falta de atención profesional del parto. La causa de muerte de estos niños fue la complicación respiratoria. 
El único caso de bronquiolitis falleció por complicación parenquimatosa pulmonar e insuficiencia respiratoria consecutiva.

Por último se registró un caso al cual se considera necesario una breve referencia: se trata de un lactante de 1 año 4 meses con antecedentes de bronquitis recidivante acompañada de marcada sialorrea desde los 6 meses de edad. Por estos hechos se sospechó la posibilidad de un cuerpo extraño el que fue confirmado por bronco y esofagoscopía. A consecuencia de la larga permanencia de este cuerpo extraño (chupete de entretención) se originó fístula traqueoesofágica que llevó a un progresivo deterioro del estado nutricional del paciente, lo que sumado a bronconeumonía por aspiración determinaron su muerte.

Infecciones generalıadas. La mayoria correspondió a preescolares y escolares que ingresan con una sintomatología florida, en la que destaca la pericarditis. Cuatro de los 7 pacientes fallecieron dentro de las primeras 24 horas de hospitalización. Entre los agentes etiológicos se encontró staphylococo dorado y Gram negativo.

Patología infeccusa del Aparato Digestivo

Tres de los cuatro pacientes corresponden a niños portadores de hepatitis que se derivaron en forma tardía, cuando las posibilidades de sobrevida eran remotas.

El último caso se refiere a un lactante desnutrido con diagnóstico de salmonellosis $y$ antecedentes de cuadro diarreico de más de 2 meses de evolución tratado con diversos agentes quimioterapéuticos.

\section{COMENTARIO}

En una visión inicial sobre el total de necropsias que representa el $29,1 \%$ de los fallecidos, llama la atención el alto porcentaje de niños del sexo masculino. Esto podría conducir erróneamente a la conclusión de una mayor mortalidad en el sexo masculino. Sin embargo, la distribución por sexo entre el total de ingresos y fallecidos no demuestra diferencia significativa. (Fig. 9).
DISTRIBUCION POR SEXO ENTRE INGRESOS Y FALLECIDOS AÑOS 1972-1974. UNIDAD INFECCIOSO. H.C.R.C.

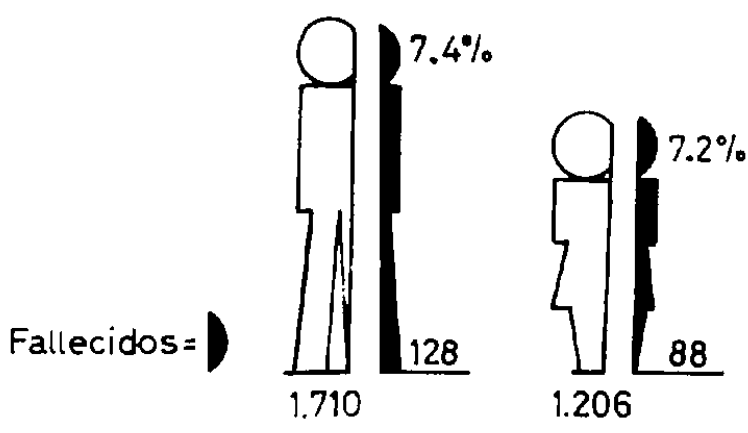

Fig. 9

Es decir, el riesgo de muerte parece ser el mismo para ambos sexos.

Cabe destacar que la distribución por sexo del total de ingresos es mayor para el grupo de varones, lo que nos podría sugerir que el varón tiene mayor riesgo de enfermar. (Fig. 10).

\section{DISTRIBUCION POR SEXO DEL TOTAL DE NIÑOS HOSPITALIZADOS ENTRE 1972-1974. UNIDAD INFECCIOSO.}

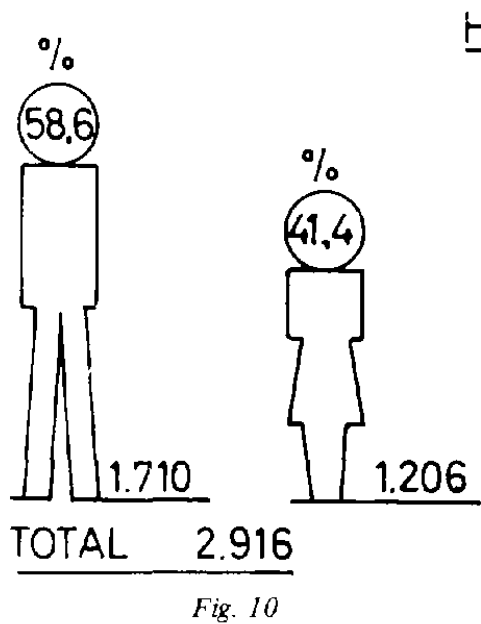

Se encontró que la mayor mortalidad está en el grupo de lactantes y de éstos, más de la mitad presentaba déficit ponderal. Como es sabido, este grupo etario posee condiciones inmunológicas $\mathrm{y}$ anatómicas que lo hacen particularmente susceptible a contraer este tipo cie enfermedades y sufrir sus complicaciones, lo que sumado a las precarias condiciones nutricionales determinan la gravedad con que cursa la patología infecciosa. 
Analizando el tiempo de estada, que muestra que un $70 \%$ de los decesos se producen antes de los 4 días del ingreso, se confirma, una vez más, la violencia y gravedad que la patología infecciosa adquiere en la niñez y permite señalar que la oportunidad en la consulta y derivación, así como el manejo inmediato, son vitales para lograr un tratamiento adecuado que dé reales posibilidades de sobrevida a estos casos graves.

La particular severidad de los procesos infecciosos del sistema nervioso representan de por sí un riesgo de muerte, que en el presente estudio fue del orden del $40 \%$ y constituyó la primera causa de muerte. La meningitis supurada es la patología específica más importante en este grupo. El grupo más afectado es el de lactantes, en el cual el diagnóstico debe plantearse ante la sospecha clínica realizando de regla una punción lumbar, ya que en ellos es poco sintomática inicialmente. Llamó la atención la gran frecuencia de otitis encontrada en el examen anatomopatológico, pudiendo haber sido ésta la puerta de entrada.

En la meningoencefalitis se debe llamar la atención sobre los cuidados de enfermería y kinesiterapia, pilares fundamentales en el buen manejo de estos pacientes, para así evitar la muerte por bronconeumonía secundaria como se comprobó en nuestro material.

La meningitis tuberculosa es una afección grave en la que sin un diagnóstico oportuno la sobrevida no vá más allá de las cuatro semanas; por ello se debe ser muy acucioso en su pesquisa. Esto se demuestra ampliamente en esta casuística, dado que en todos el diagnóstico se efectuó en forma tardía.

Dentro de la patologia del aparato respiratorio, el cuadro más importante de nuestro medio está representado por sarampión y sus complicaciones, especialmente en el año 1974 en que hubo una epidemia que determinó 440 ingresos por esta causa.

En la patología infecciosa del aparato digestivo se debe considerar la hepatitis viral, cuadro clínico que puede ser manejado en cualquier centro siempre que se tenga en cuenta la necesidad de derivar los enfermos que no presenten una evolución habitual o que evidencien signos premonitores de hepatitis grave, aseveración que no se cumplió en los tres pacientes analizados

\section{CONCLUSIONES GENERALES}

1. De las consideraciones anatomoclínicas relatadas anteriormente se puede señalar, una vez más, la importancia de las comunicaciones entre el hospital base y los hospitales periféricos para incentivar y facilitar permanentemente las relaciones entre los técnicos y profesionales de la Salud con el objeto de actualizar nuevos conocimientos, cimentar las normas de trabajo cuya eficacia ya ha sido probada $y$, en general, para desarrollar toda actividad que vaya en beneficio de nuestros pacientes.

2. Así también, y dadas las condiciones de especialización y avances actuales, estimamos que todo trabajo será tanto más útil cuanto mayor coordinación haya entre el equipo de Salud de cada centro asistencial.

3. En el caso de niños que desde el comienzo presenten dificultad diagnóstica, especialmente para los médicos generales de Zona, se estima como suficiente que sepan pesquisar el caso clínico difícil y enviarlo de inmediato al hospital base.

4. Se considera indispensable la disponibilidad permanente de un Servicio de Anatomía $\mathrm{Pa}$ tológica que permita la realización de necropsias en forma sistemática.

\section{RESUMEN}

Se realiza un análisis retrospectivo de las historias clínicas $e$ informes anatomopatológicos de 63 niños fallecidos entre los años 1972 y 1974 en la Sección Infecciosos del Servicio de Pediatria del Hospital Gmo. Grant Benavente, de Concepción.

El $73 \%$ correspondió al sexo masculino

Treinta y cuatro pacientes eran menores de dos años, y de éstos 20 menores de 6 meses.

El $50 \%$ de los pacientes falleció dentro de las primeras 48 horas de hospitalización.

La mitad de los pacientes provenia de hospitales periféricos.

Se distribuyó el material por sistema; el Sistema Nervioso ocupó la primera causa de muerte y dentro de él, la meningitis supurada; luego sigue el Aparato Respiratorio, en el que la mitad de los casos corresponde a sarampiones complicados. 